\title{
N $87-26432$
}

\section{Light Emission as a \\ Solar Cell Analysis Technique}

\author{
Christiana Honsberg and Allen M. Barnett \\ University of Delaware \\ Newark, Delaware
}

\section{Solar Cells and Light Emitting Diodes}

Since solar cells and light emitting diodes first came into common use, they have largely been considered separate areas. The information determined from one was not directly applicable to the other. However, there are many similarities between solar cells and light emitting diodes (LEDs) which suggest the potential use of light emission for solar cell analysis. Solar cells and LEDs both operate in forward bias. However, solar cells absorb light to generate current whereas LEDs require injected current to generate light. In other words, light drives a solar cell into forward bias and generates energy in the form of current at a certain voltage. In contrast, injected current in a LED drives the LED into forward bias and generates energy in the form of light. The use of light emitted from the surface of the solar cell has not been widely used as a technique to analyze solar cells.

In order to determine if a solar cell would indeed emit usable light as expected, a GaAs solar cell was forward biased and examined with an infrared viewer. The light emitted from the solar cell was not uniform, even though the I-V curve of the solar cell displayed no defects.

\section{Solar Cell Background}

A solar cell, like a LED, is a semiconductor $p-n$ junction. However, the structure of the solar cell is somewhat different than a standard semiconductor diode, since the depletion region must be near the surface in order to efficiently collect photogenerated carriers. This special structure, which in practical terms implies a very thin collector, makes the solar cell vulnerable to certain defects. Most of these defects can be simply modeled and affect the I-V curve. However, the model does not distinguish between microscopic and macroscopic defects, while the I-V curve will detect only macroscopic defects. Nevertheless, microscopic defects (which can include shunt defects) can lead to device failure. Defects which lead to device failure (mostly shunts and irregularities at the junction) are the subject of this work.

\section{Light Emission}

Since light emission begins at the junction and passes through the collector to the surface, any defects in this region will affect the pattern of light emission. Two types of light emission can be used to analyze solar cells. The first, forward biased light emission is the radiative recombination of carriers and is the mechanism operating in LEDs. In order for a semiconductor to emit light in forward bias, there must be sufficient radiative recombination of carriers and 
incomplete absorption of the radiative recombination. This works well if the material has a direct band gap, and if enough carriers can be injected and radiatively recombine so that the light can be detected at the surface of the solar cell. Additionally, the collector must not be so thick that all the light is internally re-absorbed.

Forward biased light emission is strongly dependent on the threshold current density flowing through each part of the solar cell. In areas of the solar cell with a higher current density, the light will be emitted with greater intensity than in areas with a lower current density. Consequently, the overall current flowing through the device alone does not provide much information, but must be used only in conjunction with the pattern of the light emitted from the surface. The threshold current density also determines, by the diode equation, what the threshold voltage for light emission will be. Light emission is sensitive to local variations in the current and voltage that will not affect the overall I-V curve.

The reverse biased light emission is also strongly dependent on the localized current density. However, the mechanism by which current flows across the junction and by which light is emitted is quite different than that involved in forward bias light emission. Current begins to flow across the junction due to avalanche breakdown. However, avalanche breakdown does not occur uniformly across the junction, but begins where the applied voltage is higher or the voltage required to begin avalanche breakdown is lower. Consequently, current flows only through certain small areas of the junction. Due to the carrier multiplication in avalanche breakdown, large amounts of current flow through the small areas undergoing avalanche breakdown. This high current (and power) density heats the lattice to the point where the areas undergoing avalanche breakdown begin to emit black body radiation in the visible region.

\section{Mechanisms Affecting Light Emission}

A solar cell completely free from any defects should emit light uniformly across the entire surface in forward bias. However, there are several mechanisms which will cause variations in the ideal uniformity of the light. Of major importance are those due to defects which may lead to catastrophic failure. One such defect is a shunt defect. Shunt defects, which greatly reduce the power available from a solar cell, are very common and with the advent of new thin film solar cells may become even more frequent. Shunts are usually caused by punch-through of the metal grid-pattern across the very thin collector. As can be seen from the model of a solar cell, a shunt defect acts like a current divider between the shunt defect and the area of the junction without the shunt defect. At a fixed voltage, lower shunt resistance results in more current flow through the shunt, but not less across the junction unaffected by the shunt. Consequently, the total current flowing through the solar cell when light emission begins increases while the voltage present across the solar cell remains the same. The total current will be inversely proportional to the shunt resistance, while the current density flowing across the junction needed to begin light emission remains the same.

The area near a shunt defect will not emit light. Since there are no injected minority carriers in the region of a shunt, there are no carriers to recombine and cause radiative recombination. The location of the shunt affects the pattern of light emission from the surface of the solar cell. If the shunt occurs in the bulk of the material, the solar cell will behave in the way the ideal 
model predicts, and only the small area around the shunt will not emit light. Shunts in the bulk of the material may migrate to the grid pattern and cause device failure.

If the shunt occurs along the grid pattern, portions of the solar cell will be shorted out and will remain dark. If the shunt occurs along the grid line, no current will flow through the entire region from which the grid line previously collected current. In other words, the shunt on the grid bar shorts out this entire region, much the same way in which many resistors in series will be shorted if a much lower resistor is put in in parallel with some of them. Consequently, all areas of the solar cell from which the grid line collected current will remain dark. In addition, the current going through the entire device will be much larger than if a shunt is not present, or if the shunt was only in the bulk of the material. The voltage measured at the probe will be slightly higher than a solar cell without a shunt, due to current crowding at the probe. The voltage is measured only at a single point, the probe. The entire current flowing throughout the device must flow through the probe. The higher current creates a larger voltage drop vertically across the grid bar. Since the measured voltage at the probe takes into account this voltage drop, the actual voltage across the junction unaffected by the shunt is somewhat less than the measured voltage. The voltage is higher only along the path from the shunt to the bus bar. The rest of the junction has the threshold voltage present across it.

The third place a shunt can form is on the bus bar. In this case, the entire junction is shorted out. This implies only that the majority of the current flows through the shunt rather than across the junction of the solar cell. If enough current flows through the shunt, the voltage can increase until the necessary current density to begin light emission is forced across the junction. Again, as in the case with the shunt occurring on the grid line, the voltage will be higher along the the path through which the shunt current flows, due to current crowding.

A second mechanism which affects the light emitted from the surface of the solar cell is the presence of irregularities at the junction. These defects are not detectable under examination of the I-V curve, but can also lead to catastrophic failure by lowering the reverse breakdown voltage, a key parameter in nearly all applications of the solar cell. In all practical applications the solar cells are grouped into arrays, usually groups in series and these groups parallel. If shade covers a solar cell, the solar cells that are still in the sun will drive the shielded solar cell into reverse breakdown, and possibly catastrophic failure. This will reduce the power from the entire array. It can be very difficult to test for this defect. The areas containing irregularities will not emit light, or may emit light at a higher ( or lower) current.

Another mechanism which will affect the pattern of light emission from the surface of the solar cell is an non-uniformly applied voltage. The voltage may be unevenly applied due to current crowding, series resistance, or cracks.

Finally, the light may be altered by some of the light being blocked. This can be due either to dirt or to an excessively thick collector.

All these types of defects can be distinguished from each other by examining the current density needed to begin light emission and the pattern of the light emission. A shunt defect is the only type of defect which will increase the current density and possibly voltage needed to begin light emission. Any cracks can be distinguished from other defects by the fact that the 
region between a light area and a dark area is very sharply defined. Additionally, dark areas will extend to the edges of the solar cell in at least one direction. A close visual examination may reveal a crack. Dirt on the surface of the solar cell should also have sharply defined edges but in an irregular pattern and may also be visible upon a close visual examination. Light blocked by a collector that is too thick will have very fuzzy edges, and the dark areas should become slightly smaller as the current is increased. Also, cracks, dirt, and uneven collector will all lower the short circuit current. Irregularities in the junction will affect the reverse breakdown voltage of the solar cell, but not the forward characteristics. They may have either a regular shape or an irregular shape. The edges as well may appear to be slightly fuzzy.

\section{Experimental Procedure and Results}

Light emission is especially useful for detecting defects that can lead to catastrophic failure. The two major types of defects that can lead to catastrophic are shunt defects and irregularities at the junction. In order to test for shunt defects, solar cells free from shunt defects were obtained from Joseph F. Wise of the U.S. Air Force Wright Aeronautical Laboratories. Shunt defects were then induced, and based on the light emission, the location of the shunt defect was determined and the shunt removed. The presence and location of irregularities was also determined. The procedure to demonstrate this is given below.

1. The I-V curves of the solar cells were measured to insure that there were no shunt defects present.

2. The forward biased light emission was recorded to determine the presence of any irregularities.

3. The solar cell was reverse biased until avalanche breakdown occurred.

4. The light during avalanche breakdown emission was photographed to confirm the presence and location of irregularities.

5. In order to induce a shunt defect, the avalanche current through the solar cell was increased until irreversible breakdown occurred, and the light emitted was again photographed.

6. The solar cell was forward biased and the light emission photographed.

7. The I-V characteristics of the solar cell were measured.

8. The solar cell was cut were the forward and reversed biased light emission predicted the shunt to be.

9. Both halves of the solar cell were re-examined.

All the solar cells behaved very similarly except where avalanche breakdown occurred, and the value of the reverse breakdown voltage. Both depend on the presence of irregularities at the junction. 
The solar cell shown in Figs. 1 to 4 contains many irregularities at the junction, probably doping variations or dirt at the junction of the solar cell. The many dark areas in Fig $1 \mathrm{~b}$ predict the presence of irregularities. Theoretically, these irregularities should correspond to regions where the avalanche breakdown voltage is lower, and they should begin to conduct current sooner than the rest of the junction. Since the reverse bias light emission is dependent on the current density through each area, regions with irregularities should emit light in reverse breakdown. Consequently, Fig 1c confirms the prediction of Fig $1 \mathrm{~b}$ that there are many irregularities at the junction. The avalanche breakdown voltage was rather low, approximately $2.5 \mathrm{~V}$. The solar cell in Fig 1c has not suffered irreversible breakdown, only avalanche breakdown. In order to induce a shunt defect, the current in avalanche breakdown was increased until irreversible breakdown occurred. Once the shunt was induced, the resistance in this region became lower, so most of the current began to flow through the newly created shunt defect. This accounts for the greater intensity of the light being emitted at one location once irreversible breakdown occurred. Due to the heating of the grid pattern through which the shunt current flowed, some areas of the grid pattern also became shunted. The extent to which this happened depended on how long the irreversible breakdown current was flowing through the solar cell. The shunting happened first where the power density (corresponding to the current density) was the highest. Consequently, the light seemed to move from its original location to the probe. This accounts for the slightly oval shape of the light in Fig 2a. Fig 2b, like Fig. 2a, predicts that the shunt occurred on the second grid line from the left. This location of the shunt defect was confirmed by removing the region expected to contain the shunt. The area predicted to contain the shunt did indeed display severely shunted characteristics (Fig. 4b compared to Fig. $3 \mathrm{~b}$ ) while the other region regained nearly identical characteristics to those of the original solar cell. Some shunting effects are present due to heating of the bus bar, while the current is slightly lowered due to cutting damage or inaccuracies in area measurement.

The other sample shown behaved similarly except that there were fewer irregularities at the junction. The light emitted in forward bias predicts fewer irregularities. Fewer spots suffering avalanche breakdown (see Fig 6a), combined with a higher avalanche breakdown voltage (5 V) confirms the prediction of fewer irregularities. Again, the forward bias light emission and reverse bias light emission both indicated that the shunt was on the third grid bar from left. When this area was removed, half of the solar cell displayed normal I-V characteristics ( Fig. 7a compared to 8a) while the half which was predicted to contain the shunts, showed severely shunted characteristics (Fig. 7b compared to Fig. 8b).

\section{Conclusion}

Solar cells and light emitting diodes are similar devices. A solar cell, if under light, can charge a battery, while the battery in turn can cause the solar cell to emit light like an LED. Since the solar cell can be used as two separate devices, it can be used to test itself.

Two types of light emission, forward bias and revrese bias, can be used to test the solar cell. Defects which are not readily apparent when examining the I-V curve (but can lead to device failure) can be detected using light emission. Two such common defects are irregularities at the junction and shunt defects. Both forward and reverse bias light emission can detect these types of shunts. Other, grosser defects are also readily apparent using light emission. These can include shunt defects, cracks, dirt, and an excessively thick collector. By examining the I-V 
characteristics of the solar cell, and the current density needed to begin light emission, localized information about the solar cell can be obtained and the type of defect can be determined.

Further work would include investigation into the nature of the irregularities that lower the reverse breakdown voltage. In addition, quantification of this method can lead to the development of a useful quality assurance procedure which can evaluate and predict the performance of solar cells.

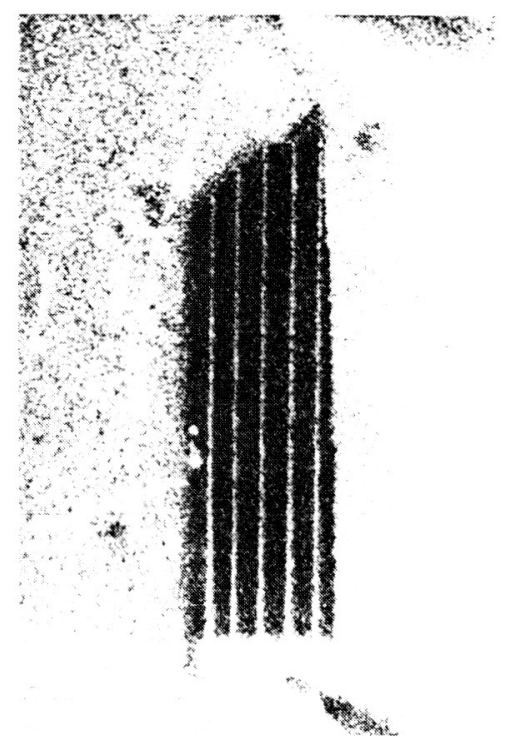

a)

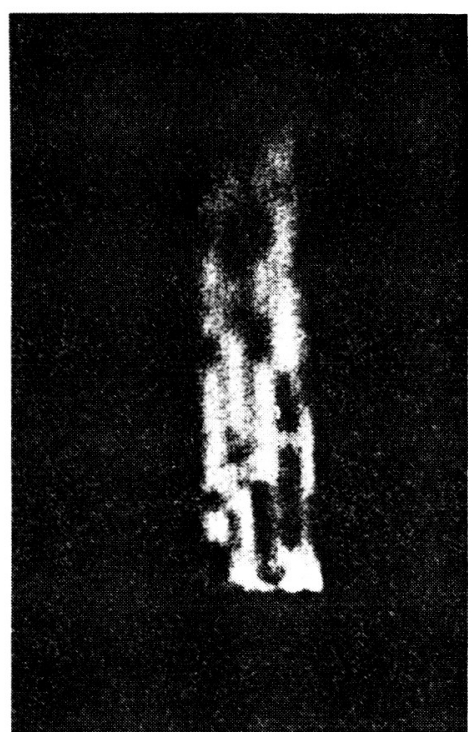

b)

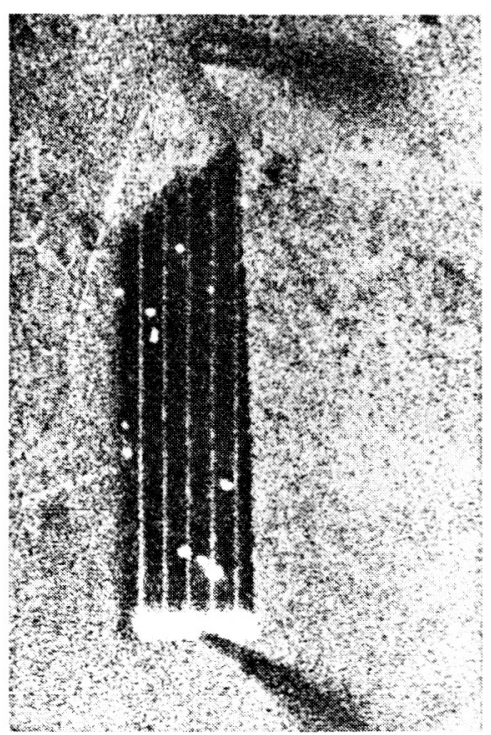

c)

Figure 1 Sample h332d a) The solar cell with no bias present across it. b) The forward bias light emission before a shunt was induced. The dark rectangle at the bottom is the bus bar. The faint vertical lines are the grid lines. The irregular dark areas correspond to irregularities at the junction. c) Reverse bias light emission before the shunt defect was introduced. The white areas are emitting light. As the theory predicted, the dark areas in b) and the light areas in c) are in similar locations. 


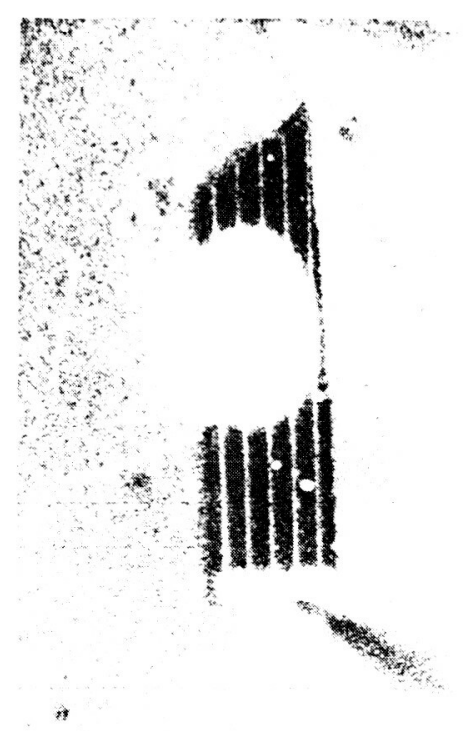

a)

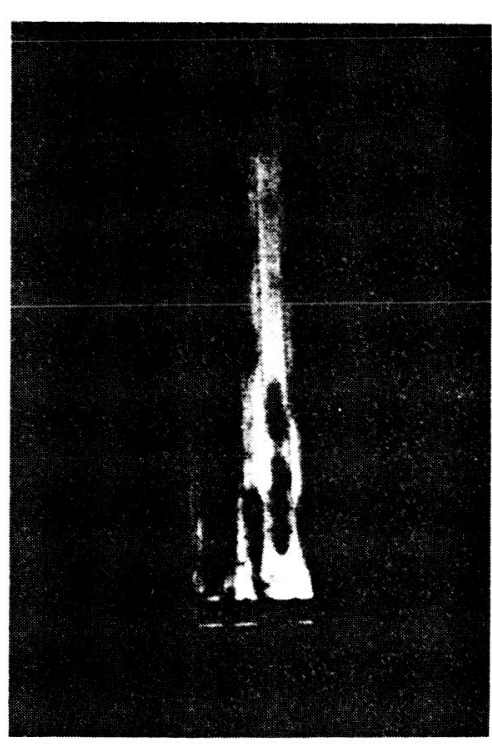

b)

Figure 2 Sample h332d a) The reverse bias light emission when the shunt was induced. The light emission appears to be oblong because the shunt is beginning to burn its way to the probe. b) The forward bias light emission after the shunt was induced. The light emission predicts the shunt to be on the second grid bar from the left. The shunt is further down than Fig. 1c suggests because the shunt continued to move towards the probe after the picture was taken.

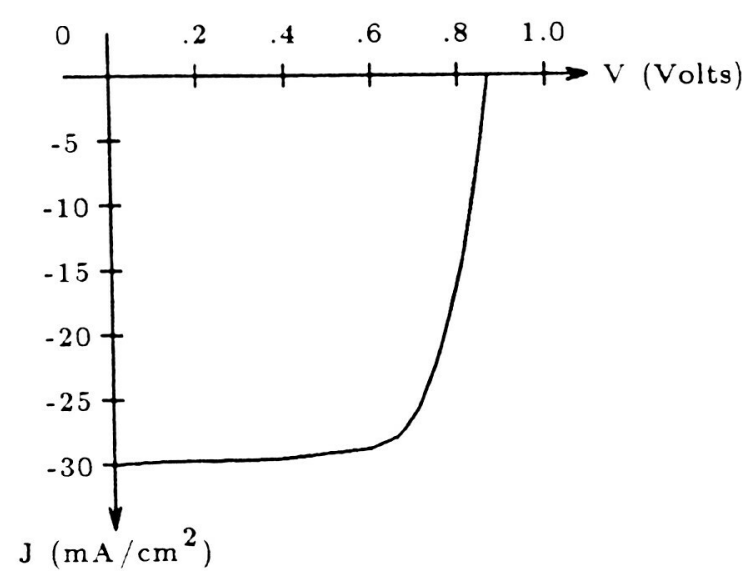

a)

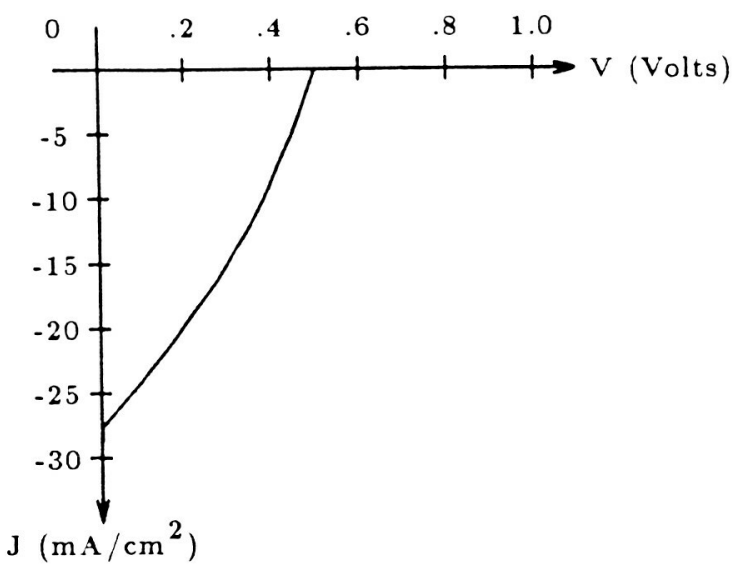

b)

Figure 3 Sample h332d a) The I-V curve of the solar cell before the shunt was induced. b) The I-V curve of the solar cell after the shunt was induced. 


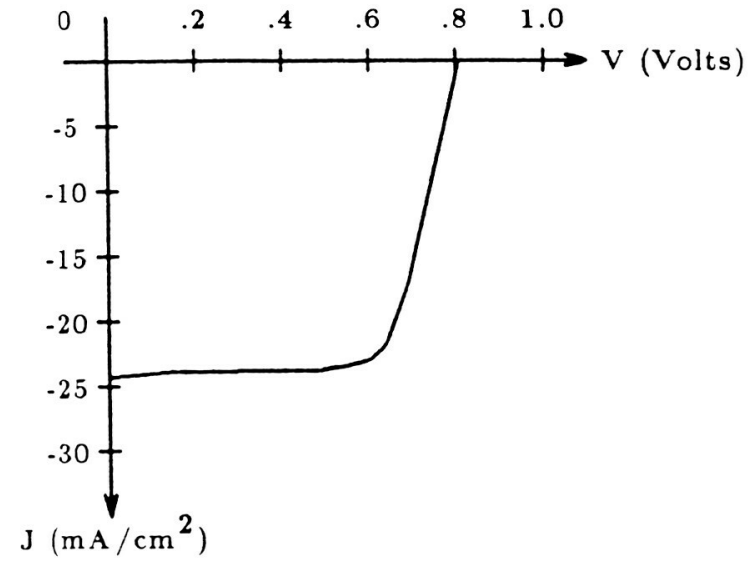

a)

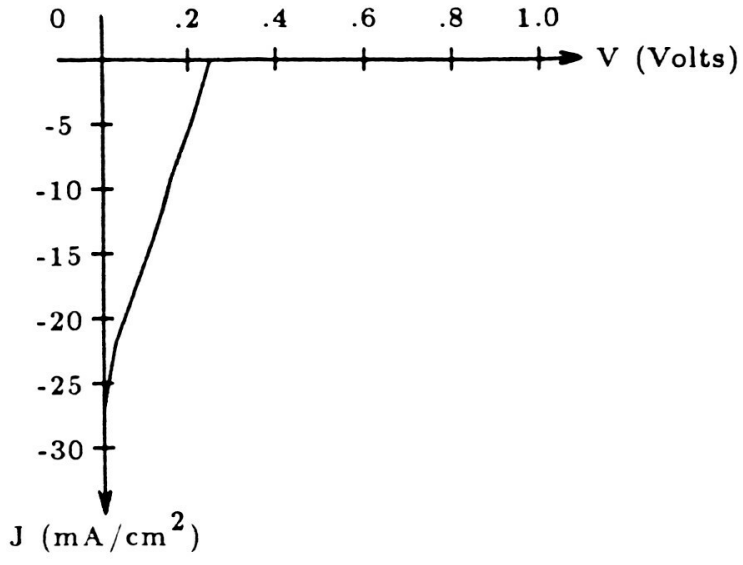

b)

Figure 4 Sample h332d a) The I-V curve of the area predicted not to contain the shunt. The curve is not identical to that in Fig. 3a due to heating of the bus bar and cutting damage. b) The I-V curve predicted to contain the shunt.

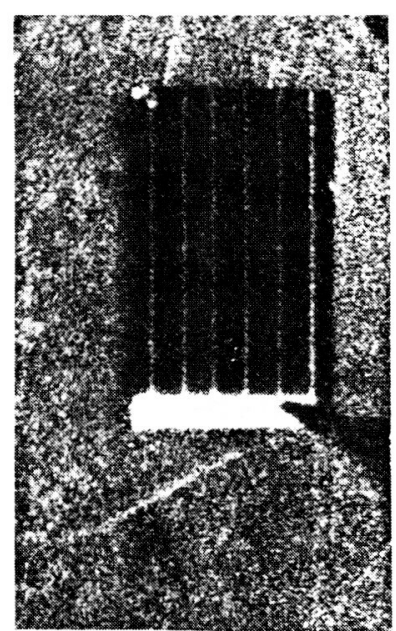

a)

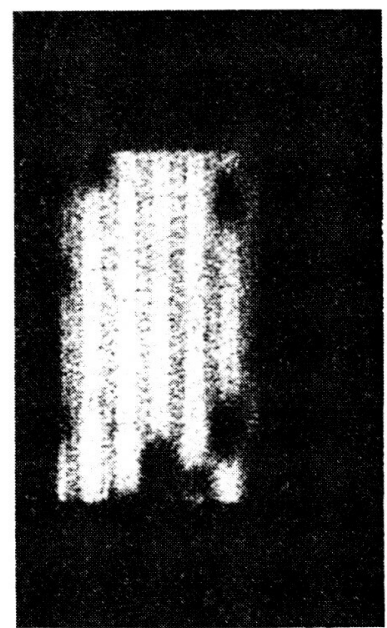

b)

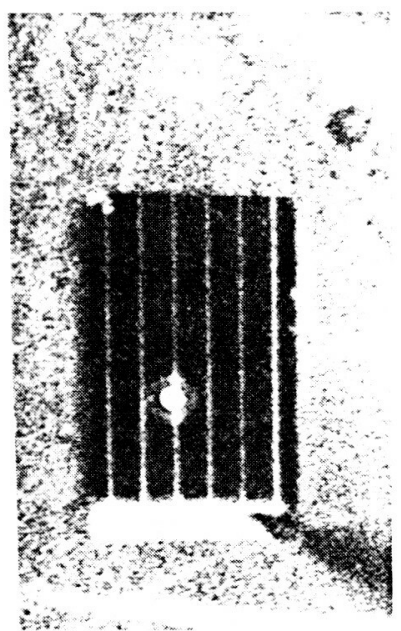

c)

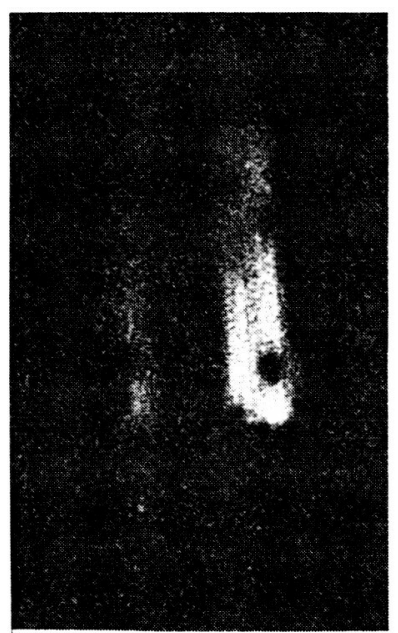

d)

Figure 5 Sample h339d a) The solar cell without any applied bias. b) The in forward bias light emission. The light emission is fairly uniform. c) The reverse bias light emission. Since the light from forward bias light emission is fairly uniform, there should be few areas emitting light in reverse bias. In this sample, only one area underwent avalanche breakdown, so the reverse bias light emission before and after the shunt was introduced will be the same. d) The forward bias light emission. The shunt was predicted to be on the third grid bar from the left, near the bottom of the solar cell. 


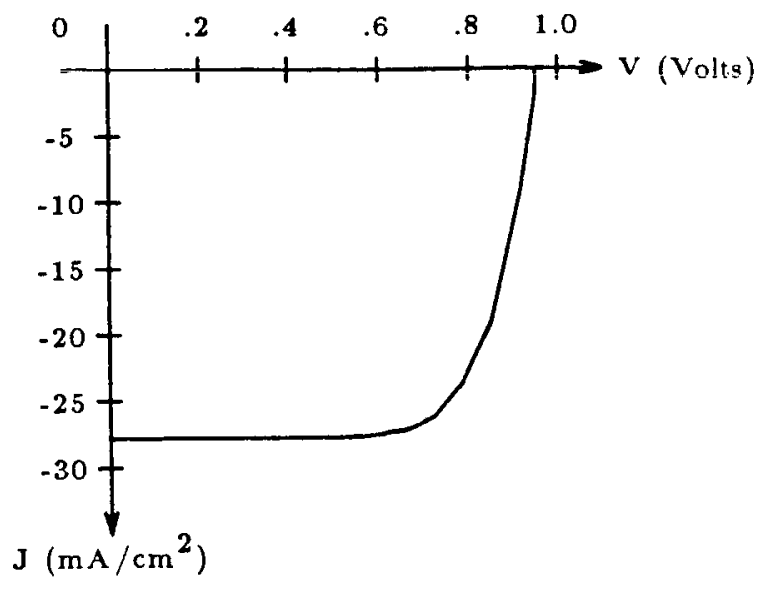

a)

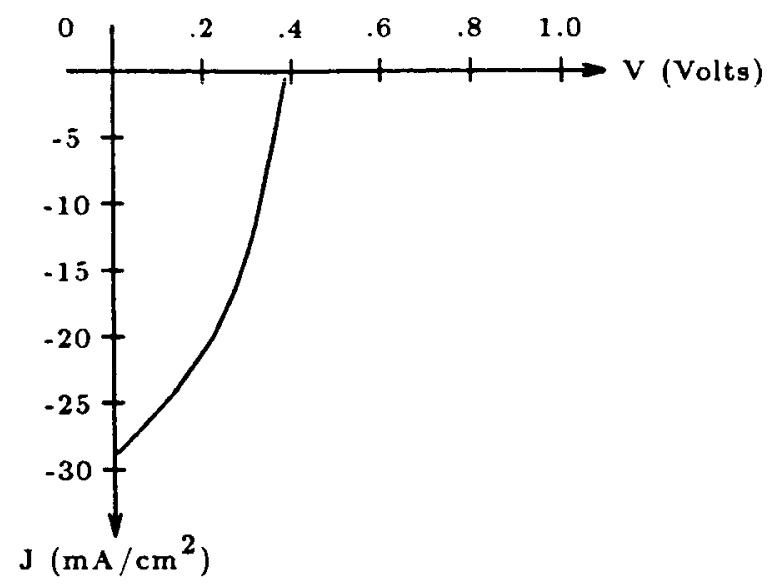

b)

Figure 6 Sample h339d a) The I-V curve of the solar cell predicted to contain the shunt. b) The I-V curve after the shunt was induced.

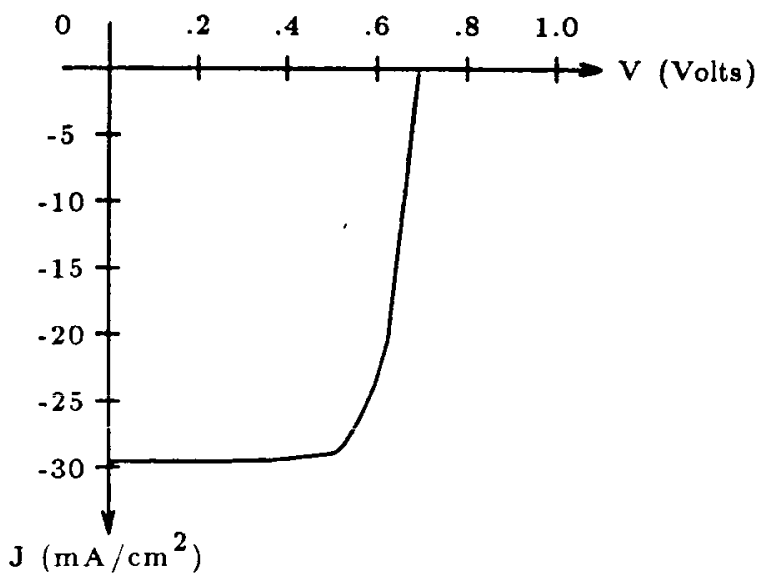

a)

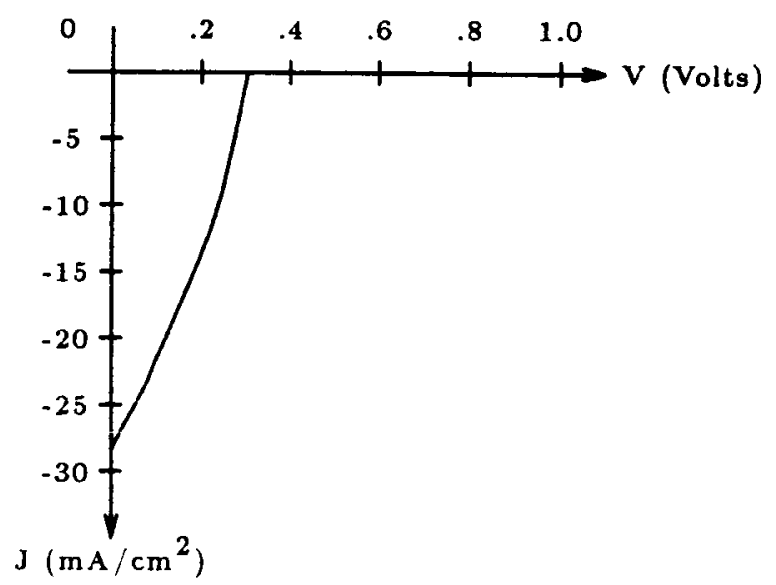

b)

Figure 7 Sample h339d a) The I-V curve of the area predicted not to contain the shunt. b) The I-V curve of the area predicted to contain the shunt.

$$
c-3
$$

